\title{
Metabolic Disturbances and the Intestinal Microbiome
}

\author{
Luigi F Meneghini, MD, MBA
}

Professor of Internal Medicine, UT Southwestern Medical Center; Executive Director, Global Diabetes Program for the Parkland Health

and Hospital System, Dallas, Texas, US

\section{Summary}

The gut microbiome, which can be affected by dietary and other "environmental" conditions, appears to have an important role in health and disease. A better understanding of the impact of changes in our gut microflora, as well as the determinants of these changes, represents an opportunity to address a number of acute and chronic disease conditions through novel mechanisms and therapeutic approaches.

\section{Keywords}

Microbiome, insulin resistances, obesity

Disclosure: Luigi F Meneghini, MD, MBA, has no conflicts of interest to declare. No funding was received in the publication of this article.

Open Access: This article is published under the Creative Commons Attribution Noncommercial License, which permits any noncommercial use, distribution, adaptation, and reproduction provided the original author(s) and source are given appropriate credit.

Received: March 4, 2015 Accepted: March 16, 2015 Citation: US Endocrinology, 2015: 11(1):34-5 DOl: http://doi.org/10.17925/USE.2015.11.1.34

Correspondence: Luigi F Meneghini, MD, MBA, 5323 Harry Hines Blvd, MC-8857, Dallas TX 75390-8857, US. E: luigi.meneghini@utsouthwestern.edu

Recent advances in high-throughput metagenomic sequencing have provided a substantial body of knowledge regarding the gut microbiome and its potential relationship to disease. ${ }^{1}$ Although the search for genetic explanations of chronic disease, such as diabetes and insulin resistance, continues, there exists growing interest in the epigenetic determinants of such conditions. The human genome consists of around 23,000 genes, whereas the gut microbiome contributes to approximately 3 million genes, representing over $99 \%$ of our genetic makeup. ${ }^{2}$ The specific composition of gut microbiota and their respective metabolites appears to be influenced by dietary intake and can either positively affect the host, such as through the production of butyrate, or contribute to toxicity, such as through the absorption of lipopolysaccharides. These changes appear to be, in part, associated with changes in the intestinal microflora composition and diversity. ${ }^{3}$ For example, in type 2 diabetes, some studies have reported an increase in certain species of Firmicutes and a decrease in certain Bacteroidetes species, whose phyla collectively account for more than $90 \%$ of the gut microbiota. ${ }^{4}$

Experiments in rodents have demonstrated an association between the gut microbiome and body weight, as well as insulin resistance and the development of diabetes. For example, germ-free mice gain less weight on a western high-fat diet than conventional mice, but transplantation of fecal material from the gut of conventional or obese animals to that of germ-free rodents induces weight gain and insulin resistance. ${ }^{5}$ In addition, creating pseudo-germ-free mice via treatment with specific antibiotic "cocktails" to wipe out intestinal microflora can prevent the development of obesity in mice fed a high-fat diet, including leptin-deficient ob/ob mice. ${ }^{2}$ Initial experiments in humans appear to support a causative role for the microbiome with respect to obesity and the metabolic syndrome. Bacterial production of endotoxins, such as liposaccharide (LPS) from Gram-negative bacteria, has been implicated in obesity. Obese humans and rodents tend to have greater burden of LPS-producing bacteria and higher LPS concentrations in plasma, which can be reversed with healthy changes in diet and lifestyle, leading to weight loss. ${ }^{2}$

Initial attempts to establish a causative relationship between intestinal flora and metabolic disturbances are under way. In a proof-of-concept experiment, vrieze and colleagues selected insulin-resistant individuals and randomized them to receive either an autologous microbiota infusion or an allogenic gut microbiota infusion from lean donors. ${ }^{6}$ Compared with subjects receiving autologous microbiota, the experimental group exhibited improvement in insulin sensitivity, associated with increases in levels of bacteria producing butyrate, within 6 weeks of transplantation. In another recently published series of experiments, Suez and colleagues elegantly demonstrated the causative relationship between altered intestinal flora and the development of glucose intolerance and insulin resistance after ingestion of an artificial sweetener in rodents. ${ }^{7}$ They additionally explored the causative relationship between noncaloric artificial sweeteners and glycemic parameters by having seven healthy volunteers ingest the US Food and Drug Administration (FDA)'s maximum acceptable daily intake of saccharin for a week. Four of the subjects developed worsening glycemic responses, as measured by glucose tolerance testing and continuous glucose monitoring, associated with substantial changes in their microbiome composition. Stool transfer from a sample of these patients after 7 days of exposure to saccharin-induced glucose intolerance in germ-free mice recipients compared with no effect from stools collected before saccharin exposure.

A better understanding of the role of the gut microbiome in health and disease, and its effects on response or resistance to treatment, whether 
dietary, lifestyle, or pharmacologic, represents an exciting opportunity to affect health through novel pathways and pathophysiology. These preliminary and certainly provocative observations in the field of obesity and metabolic disturbances might be but harbingers of what might evolve in our quest to develop more targeted approaches to these challenging health matters.
1. Claesson MJ, O'Toole PW, Evaluating the latest high-throughput molecular techniques for the exploration of microbial gut communities, Gut Microbes, 2010;1:277-8.

2. Zhao $L$, The gut microbiota and obesity: from correlation to causality, Nat Rev Microbiol, 2013;11:639-47.

3. Le Chatelier E, Nielsen T, Qin J, et al., Richness of human gut microbiome correlates with metabolic markers, Nature,
2013;500:541-6.

4. Eckburg PB, Bik EM, Bernstein CN, et al., Diversity of the human intestinal microbial flora, Science, 2005;308: $1635-8$

5. Backhed $\mathrm{F}$, Ding $\mathrm{H}$, Wang $\mathrm{T}$, et al., The gut microbiota as an environmental factor that regulates fat storage, Proc Natl Acad Sci U S A, 2004;101:15718-23.
6. Vrieze A, Van Nood E, Holleman F, et al., Transfer of intestinal microbiota from lean donors increases insulin sensitivity in individuals with metabolic syndrome, Gastroenterology 2012;143:913-6.e7.

7. Suez J, Korem T, Zeevi D, et al., Artificial sweeteners induce glucose intolerance by altering the gut microbiota, Nature, 2014;514:181-6. 